\title{
Snapping knee due to the biceps femoris tendon treated with repositioning of the anomalous tibial insertion
}

\author{
Hideki Date $\cdot$ Kazue Hayakawa $\cdot$ Kenji Nakagawa • \\ Harumoto Yamada
}

Received: 13 July 2011/ Accepted: 8 November 2011/Published online: 23 November 2011

(c) The Author(s) 2011. This article is published with open access at Springerlink.com

\begin{abstract}
Snapping knee associated with biceps femoris tendon that caused lateral knee pain is reported. The long head of the biceps femoris tendon had an anomalous tibial insertion in addition to the direct arm and anterior arm on the fibular head. Resection of both the anomalous tibial insertion and the anterior arm was necessary to resolve the snapping.

Level of evidence IV.
\end{abstract}

Keywords Snapping knee - Biceps femoris tendon · Anomalous insertion - Surgical treatment

\section{Introduction}

Snapping knee due to the biceps femoris tendon can be caused by tendon injury or various anatomical pathologies, including anomalous insertion of the long head of the biceps femoris tendon and fibular head deformity. In a case of symptomatic snapping, operative management may be necessary [1-9, 11]. In previous cases associated with anomalous tibial insertion of the long head of the biceps femoris tendon reported by Kissenberth and Wilckens [8] and Bansal et al. [3], the snapping phenomenon was resolved by repositioning of the anomalous tibial component to the posterolateral portion of the fibular head. In the present case, repositioning of the anomalous tibial insertion

H. Date $(\bowtie) \cdot$ K. Hayakawa $\cdot$ K. Nakagawa $\cdot$ H. Yamada Department of Orthopedics,

Fujita Health University School of Medicine,

1-98 Dengakugakubo Kutsukake-cho, Toyoake-city,

Aichi, Japan

e-mail: date@fujita-hu.ac.jp and anterior arm of the biceps femoris tendon to the posterolateral portion of the fibular head was necessary to resolve the snapping.

\section{Case report}

A 15-year-old male patient presented with a history of increasing snapping and pain on the lateral side of his right knee over a two-year period. There was no history of trauma, and he had not engaged in continuous athletic exercise.

On physical examination, an audible and painful snapping was elicited over the fibular head at $100^{\circ}$ knee flexion and was increased by internal rotation of the tibia. All other aspects of the knee examination were normal; however, a similar snapping was apparent for the contralateral knee, but without pain. Routine radiographs were normal. Magnetic resonance imaging and computed tomography did not demonstrate any abnormalities.

In surgical exploration, it was found that insertion of the long head of the biceps femoris tendon had divided into three tendinous components. One component was inserted on the anterolateral aspect of the proximal tibia, and the others were located on the lateral edge and posterolateral portion of the fibular head (Fig. 1). The biceps femoris tendon displaced over the fibular head during knee flexion and extension without an apparent anatomical anomaly of the fibular head. Resection of the tibial component did not resolve the snapping phenomenon. After additional resection of the anterior arm on the lateral edge, snapping was removed during knee flexion and extension. The resected components were each sutured with three stitches using size 0 Surgilon directly against the periosteum and direct arm on the posterolateral portion of the fibular head 
(Fig. 2). Immediately after the operation, the patient had relief from snapping.

\section{Discussion}

The principal finding in this case was that the insertion of the long head of the biceps femoris tendon was divided into three tendinous components. One component was inserted on the anterolateral aspect of the proximal tibia, and the others were located on the lateral edge and posterolateral portion of the fibular head. In previous cases, snapping knee associated with the biceps femoris tendon has been found to be due to anomalous insertion of the long head of the biceps femoris tendon [2, 3, 6, 8-10], fibular head deformity [1,5], tendon injury [4], and normal anatomy without trauma [13].

Terry and LaPrade [11] described the normal anatomy of the biceps femoris tendon complex in detail. The long head of the tendon divides into two tendinous components, a direct arm and an anterior arm, and three fascial components that fan out to the anterior and distal tibia. The direct arm inserts on the posterolateral edge of the fibular head lateral to the fibular styloid, and the anterior arm insertion is located along the lateral edge of the fibular head. In the present case, the two components located on the lateral edge and posterolateral portion of the fibular head were identified as the anterior arm and direct arm, respectively, and the component inserted on the anterolateral aspect of the proximal tibia was an anomalous insertion.

Kristensen et al. [9], Hernandez et al. [6], and Bagchi and Grelsamer [2] reported snapping knee due to the insertion of the entire tendon of the long head on the

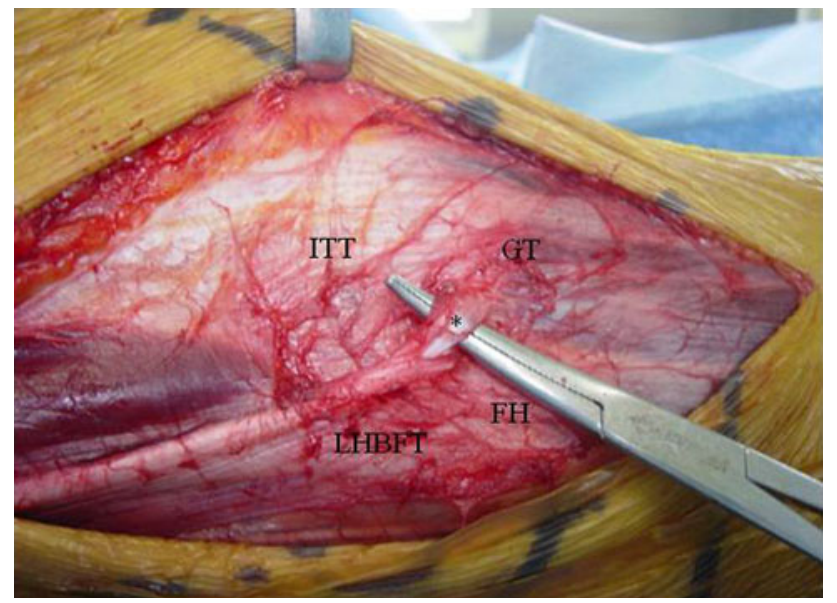

Fig. 1 Intraoperative photograph showing anomalous tibial insertion on the anterolateral aspect of the proximal tibia. *anomalous tibial insertion, ITT iliotibial tract, GT Gerdy tubercle, LHBFT long head of biceps femoris tendon, $\mathrm{FH}$ fibular head

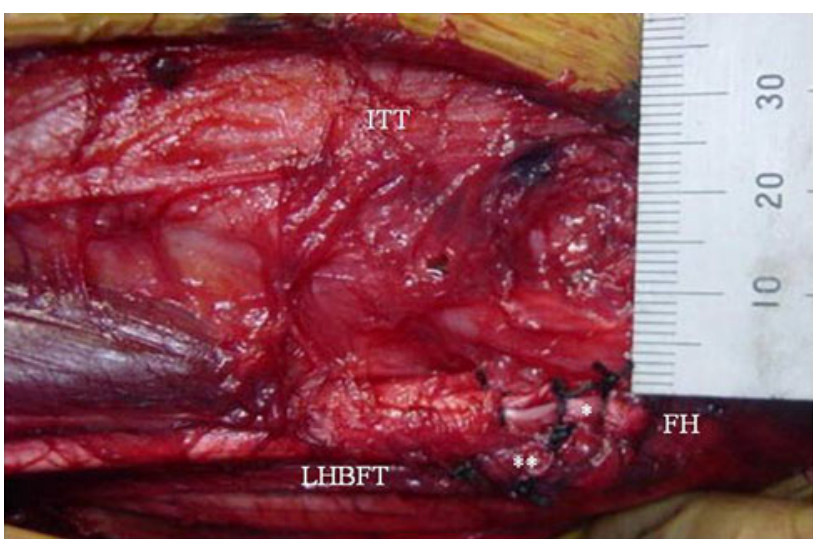

Fig. 2 Intraoperative photograph after reattaching of the anomalous tibial component and anterior arm, each sutured with three stitches on the posterolateral portion of the fibular head. *anomalous tibial insertion, **anterior arm of biceps femoris tendon, ITT iliotibial tract, LHBFT long head of biceps femoris tendon, $F H$ fibular head

anterolateral aspect of the proximal tibia. Adequate partial resection of the fibular head resolved the symptoms [2, 9], and reinsertion of the entire tendon on the posterolateral portion of the fibular head gave a good outcome [6]. Lokiec et al. [10] described the insertion of the entire tendon of the long head on the anterior portion of the fibular head. Resolution was accomplished by reinsertion of the anterior half of the tendon on the posterolateral portion of the fibular head. Kissenberth and Wilckens [8] and Bansal et al. [3] reported cases with location of part of the long head on the anterolateral aspect of the proximal tibia. Repositioning of the anomalous tibial insertion on the posterolateral portion of the fibular head resolved the symptoms in both cases. In cases with fibular head deformity, Fung et al. [5] described a case caused by an exostosis, and Bach and Minihane [1] found that a prominent fibular head elicited snapping. Snapping in these cases was resolved by resection of the exostosis and partial resection of the fibular head, respectively. Bernhardson and LaPrade [4] reported three cases of direct arm injury with normal anatomy, in which repair of the injured direct arm resolved the symptoms. Vavalle and Capozzi [13] described a case with normal location of the long head on the fibular head that presented with a snapping phenomenon. Partial resection of the fibular head resolved the symptoms.

The present case involved an anomalous tibial component of the biceps femoris tendon, as also seen in previous cases [3, 8]. However, in contrast to the previous cases, resection of the anomalous tibial insertion alone did not remove snapping and additional resection of the anterior arm was necessary to resolve the snapping. Therefore, snapping may have been caused by anterior arm insertion of the biceps femoris tendon, in addition to anomalous tibial insertion. 
In a case of snapping knee due to semitendinosus and gracilis tendons, in contrast to the biceps femoris tendon, Karataglis et al. [7] reported a good outcome without functional loss after tenotomy and resection of the tendons, which are also hamstrings and medial stabilizers of the knee. The biceps femoris is the strongest hamstring responsible for knee flexion and external rotation and plays an important role in knee stabilization, making it necessary to repair the resected tendons firmly for the prevention of tendon rupture. In repair of the biceps femoris tendon, Kissenberth and Wilckens [8] and Bernhardson and LaPrade [4] described reattachment of resected or injured tendons on the fibular head using bone suture anchors. Recently, Tsai et al. [12] have described a strong repair technique for a ruptured biceps femoris tendon using a suture anchor and reverse fascia flap. In the present case, the resected components were sutured with stitches only directly against the periosteum and direct arm on the posterolateral portion of the fibular head, whereas firm fixation of the resected tendons using a suture anchor may have been preferable.

\section{Conclusions}

Operative management for this condition primarily includes the repositioning of the snapping tendon and partial resection of the fibular head. In a case with no fibular head deformity, resection of the snapping tendon is technically straightforward and repositioning on the posterolateral fibular head should resolve the symptoms of pain and snapping effectively.

Open Access This article is distributed under the terms of the Creative Commons Attribution Noncommercial License which permits any noncommercial use, distribution, and reproduction in any medium, provided the original author(s) and source are credited.

\section{References}

1. Bach BR Jr, Minihane K (2001) Subluxating biceps femoris tendon: an unusual case of lateral knee pain in a soccer athlete. Am J Sports Med 29:93-95

2. Bagchi K, Grelsamer RP (2003) Partial fibular head resection for bilateral snapping biceps femoris tendon. Orthopedics 26:1147-1149

3. Bansal R, Taylor C, Pimpalnerkar AL (2005) Snapping knee: an unusual biceps femoris tendon injury. Knee 12:458-460

4. Bernhardson AS, LaPrade RF (2010) Snapping biceps femoris tendon treated with an anatomic repair. Knee Surg Sports Traumatol Arthrosc 18:1110-1112

5. Fung DA, Frey S, Markbreiter L (2008) Bilateral symptomatic snapping biceps femoris tendon due to fibular exostosis. J Knee Surg 21:55-57

6. Hernandez JA, Rius M, Noonan KJ (1996) Snapping knee from anomalous biceps femoris tendon insertion: a case report. Iowa Orthop J 16:161-163

7. Karataglis D, Papadopoulos P, Fotiadou A, Christodoulou AG (2008) Snapping knee syndrome in an athlete caused by the semitendinosus and gracilis tendons. A case report. Knee 15:151154

8. Kissenberth MJ, Wilckens JH (2000) The snapping biceps femoris tendon. Am J Knee Surg 13:25-28

9. Kristensen G, Nielsen K, Blyme PJH (1989) Snapping knee from biceps femoris tendon. A case report. Acta Orthop Scand 60:621

10. Lokiec F, Velkes S, Schindler A, Pritsch M (1992) The snapping biceps femoris syndrome. Clin Orthop Relat Res 283:205-206

11. Terry GC, LaPrade RF (1996) The biceps femoris muscle complex at the knee. Its anatomy and injury patterns associated with acute anterolateral-anteromedial rotator instability. Am J Sports Med 24:2-8

12. Tsai TY, Yang YS, Yeh YK, Chuang FK, Liu CW, Pan RY (2010) A new operative treatment for chronic biceps femoris tendon avulsion. Knee Surg Sports Traumatol Arthrosc 18:787789

13. Vavalle G, Capozzi M (2010) Symptomatic snapping knee from biceps femoris tendon subluxation: an unusual case of lateral pain in a marathon runner. J Orthopaed Traumatol 11:263-266 